\title{
Aerodynamic shape optimization of axial flow fan nose cones
}

\author{
R. W. Derksen ${ }^{1} \&$ J. R. Bender ${ }^{2}$ \\ ${ }^{I}$ Department of Mechanical and Manufacturing Engineering, \\ University of Manitoba, Canada \\ ${ }^{2}$ Airline Analysis, Boeing Commercial Airplanes, USA
}

\begin{abstract}
The purpose of this work is to improve the efficiency of large scale axial flow fans through the introduction of a more uniform velocity distribution over the fan blades. This improved velocity distribution being realized through the use of an aerodynamically optimized inlet cone. The procedure for optimizing the inlet cone uses existing aerodynamic optimization methods programmed into a completely self contained FORTRAN program. The type of optimization algorithm used here is the use of design optimization to solve an inverse design problem. The different modules of the program include a surface vorticity panel method flow solver, a Bezier curve surface definition routine and a minimization method. Three different minimization methods were tested to determine the most appropriate one, this being the downhill simplex method in multidimensions. Many different sized fans and inlet cones were tested, with two different types of optimized inlet cones being discovered. Short inlet cones typically make use of a very blunt inlet cone with a slight hump or rise above the hub radius. Longer inlet cones make use of a more curved inlet cone with no hump. It was also found from this work that the relatively simple methods used can provide an adequate modeling of the problem and a reasonable solution.

Keywords: axial flow fans, aerodynamic shape optimization, inverse design.
\end{abstract}




\section{Introduction}

The main objective for this work is to increase the efficiency of industrial and commercial fans and turbines by introducing a more uniform flow at the inlet plane to the blading than normally exists. An example of an axial flow fan and its components is shown in Figure 1 below.

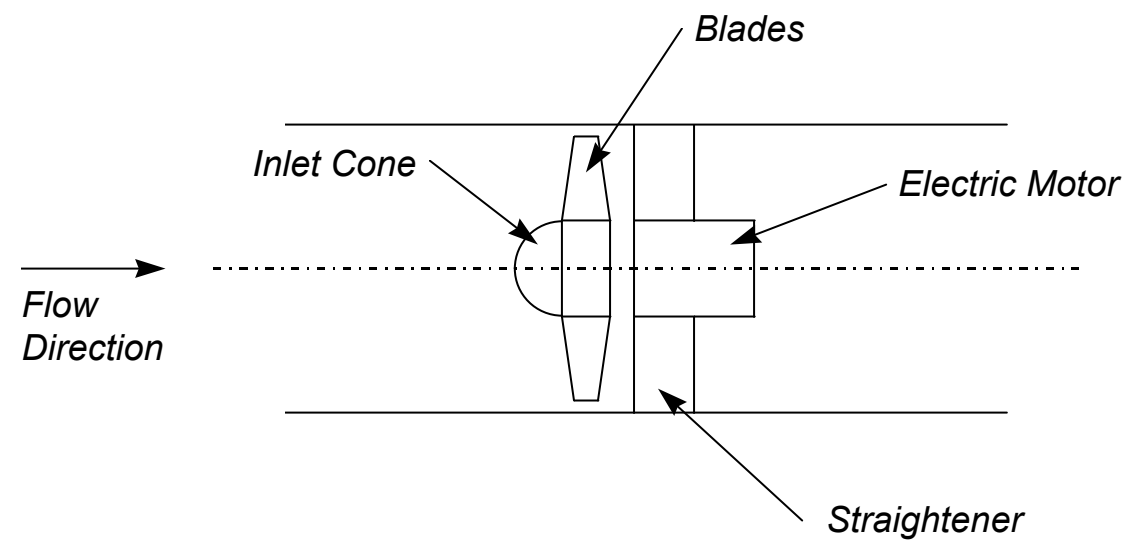

Figure 1: An example of an axial flow fan.

One of the more useful methods is the use of design optimization to solve an inverse design problem. This method makes use of a prescribed final condition such as an optimal velocity distribution or pressure distribution and uses one of the many minimization or maximization methods to search for the proper geometry to provide the desired distribution.

\subsection{The flow optimization process}

Three main program modules are required for an aerodynamic shape optimization program. The modules are: the flow solver, shape definition and minimization algorithms. A full discussion of the program details and issues that form its development are given in Bender [1].

The flow solver used in this research was an inviscid potential flow model, a surface vorticity model based on Martensen method, to model the flow in the duct and around the inlet cone. The problem is axisymmetric, as all objects involved will be either bodies of revolution, ducts or annuli. The equations used to model axisymmetric flow are different from the standard 2-dimensional equations. This work is based on the work of Lewis [2] who provides a derivation and explanation of the necessary equations and programming issues that arise.

The shape of the inlet cone was parameterized using Bezier curves which are specified by a set of control points that "steer" the curve towards them. The curve only passes through the two end points, however it does remain in the 
convex hull (the polygon defined by the control points) of the control points. The function of each control point is to bend the curve towards it as the curve gets near. The use and theory of Bezier curves is thoroughly discussed in Newman and Sproull [3] and Paulidis [4] and will not be elaborated on here.

Three separate minimization techniques were used to solve the current problem: successive iteration of Brent's method in one-dimension, the downhill simplex method in multidimensions and simulated annealing. The first two methods are known as "hill climbing" techniques. That is, they simply follow the local landscape until they reach a minimum. Simulated annealing is a more complicated method that searches the solution domain for the global minimum.

One-dimensional minimization, in particular Brent's method, is very well understood and the theory can be found in most introductory numerical analysis text books such as Press et al. [5]. Brent's method is one of the simplest types of minimization methods, a "hill climbing" technique. One of the drawbacks of Brent's method is that the algorithm must have a minimum bracketed before it is started. This can be accomplished through the use of a bracketing routine such as the one found in Press et al. [5] and used in this project. Brent's method can be used to solve a multidimensional minimization problem through the use of successive iterations. Each design variable is in turn used as the variable in the function to be minimized while all other design variables are held constant.

The downhill simplex method in multidimensions was introduced by Nelder and Mead [6]. The algorithm is set up to locate a minimum of a function of more than one independent variable. This method is still a "hill climbing" method and therefore does not guarantee that a global minimum will be found. The downhill simplex method makes use of a simplex to find its way "downhill" to a minimum. A simplex in $\mathrm{N}$ dimensions consists of $\mathrm{N}+1$ points or vertices and the lines that connect these vertices. For example, in two dimensions (two independent variables) the simplex is a triangle. Once the initial simplex is defined the algorithm continuously modifies it in such a way as to allow it to "crawl" downhill to a minimum where the program is terminated. The simplex is moved downhill through the use of three basic steps: reflection, expansion and contraction of the simplex. The most common step is the reflection, where the "highest point" is moved through the opposite face of the simplex to a lower point. The simplex is also allowed to expand in one direction to allow it to move more quickly down a valley floor. The simplex can also contract itself around one point or a minimum.

Simulated annealing is a technique that uses an analogy with thermodynamics to solve minimization problems. Simulated annealing has the attractive feature that it can search out a global minimum even when it is located amongst many local minimums. The thermodynamic analogy uses the way in which a metal cools and anneals to its lowest energy level. There are four basic elements that must be present for the algorithm to work: an objective function $\mathrm{E}$ (analogous to energy) that is to be minimized, a control parameter $\mathrm{T}$ (analogous to temperature), an annealing schedule for the reduction of $\mathrm{T}$ and a random number generator to provide a random search of the design space. There are several different variations of simulated annealing in the literature, but the variation 
chosen was found in Press et al. [5] and works as follows. The system state is described by a simplex of $\mathrm{N}+1$ points as in the downhill simplex method in multidimensions, and the simplex moves are the same as those described previously. At any temperature $\mathrm{T}$ the simplex scales itself to the approximate size of the region that can be searched at that temperature. The region is then randomly searched. The routine will always accept a downhill step but will also sometimes accept an uphill step. This is known as the Metropolis procedure and is what allows the algorithm to escape from a local minimum in favour of a global minimum. Therefore, if the temperature is reduced sufficiently slowly it is likely that the simplex will shrink into the global minimum.

Several different objective functions were considered for use in the program. For the sake of simplicity the following function was chosen:

$$
F(x)=\left|\frac{U_{\text {max }}}{U_{\text {min }}}-1\right|
$$

$U_{\max }$ and $U_{\min }$ are the maximum and minimum velocities on the blade inlet velocity distribution. It is easily shown that as the velocity distribution approaches uniformity this function will approach zero, and the more uniform the velocity distribution the lower the objective function value will be.

\section{Problem definition}

The tests used to compare the three different minimization methods were done using a single hub-to-tip ratio, $h / R_{T}=0.25$, fan, with non-dimensional hub lengths, $L / R_{T}$, of: $0.125,0.25,0.500 .75$, and 1.0 .

Tests were undertaken to determine the effect of hub-to-tip ratio on the solution.

In order to determine the effect of the hub-to-tip ratio a number of tests were run using three different $h / R_{T}: 0.25,0.5$, and 0.75 . For each hub-to-tip ratio five different inlet cone lengths were tested. These lengths were the same as used for the tests on the minimization methods

Table 1: $\quad$ Number of function calls required for stopping criteria of 0.001 .

\begin{tabular}{|c|c|c|c|c|c|}
\hline Method & $0.25 \mathrm{~L} / R_{T}$ & $0.50 \mathrm{~L} / R_{T}$ & $1.0 \mathrm{~L} / R_{T}$ & $1.5 \mathrm{~L} / R_{T}$ & $2.0 \mathrm{~L} / \mathrm{R}_{T}$ \\
\hline Original 1-D & 147 & 153 & 146 & 288 & 147 \\
\hline Multi - D & 43 & 33 & 97 & 91 & 160 \\
\hline $\begin{array}{c}\text { Simulated } \\
\text { Annealing }\end{array}$ & 100 & 118 & 151 & 211 & 212 \\
\hline
\end{tabular}

\section{Results}

The tests on the minimization methods were done with a very fine stopping condition of 0.001 . This value corresponds to the smallest change in the objective function that will not terminate the optimization process. Table 1 gives the number of flow solver function calls required by each method. 
The most important result that can be determined is that the downhill simplex method in multidimensions is significantly better than the other two methods. Therefore the downhill simplex method is considered to be the most efficient minimization method for this problem.

The objective function value was observed to be significantly higher for large hub-to-tip ratio fans with short inlet cone lengths. The general trend for inlet cone length is that as the cone length is increased the objective function is decreased. These two results were expected from performance data on industrial fans. As the $h / R_{T}$ is increased the efficiency of the fan unit generally decreases. Due to the fact that some fan manufacturers use an arbitrarily shaped inlet cone with a length of $0.125 R_{T}$ or less there is no efficiency data for different inlet cone lengths. However, it is an intuitive assumption that the value of the objective function used would decrease with increasing inlet cone length.

The most surprising observation was that the $0.75 \mathrm{~h} / R_{T}$ fan actually provided a lower objective function than the other two for an inlet cone length of $0.25 R_{T}$. Also, for an inlet cone length of $0.50 R_{T}$ or longer there is almost no difference in final objective function value among the three different hub-to-tip ratios. This would suggest that there is an optimum inlet cone length for each $h / R_{T}$. This optimum inlet cone length would then correspond to a point of diminishing returns where any subsequent increase in inlet cone length would not significantly decrease the final objective function value or increase the efficiency of the fan.

Figure 2 gives the final designs and streamlines for the $0.25 h / R_{T}$ fans, Figure 3 gives the final designs and streamlines for the $0.5 h / R_{T}$ fans, and Figure 4 gives the final designs and streamlines of the $0.75 h / R_{T}$ fans. There are two different classes of final shapes; the first type of shape has a distinctive hump or rise near the front of the inlet cone, while the second type is just a slight variation of the initial parabolic shape.

These figures provide examples of the first type of inlet cone shape with a distinctive hump. This type of inlet cone is characterized by a very blunt face with a distinctive hump that rises above the hub radius. This class of inlet cone shape was found mainly on the shorter inlet cones. Typically, inlet cone lengths of less than $0.75 \mathrm{~L} / R_{T}$ demonstrated this type of shape. The one exception to this is the inlet cone for the $0.75 \mathrm{~h} / R_{T}$ fan with an inlet cone length of $0.125 R_{T}$. It is unknown why this case demonstrates the second class of inlet cone shape.

The second class of inlet cone shape is classified by a very smooth curved inlet cone with a relatively large radius of curvature and by the lack of a blunt face and hump. This parabolic shape was originally expected for all inlet cone lengths. However, this type of shape was typically found on inlet cones of length $0.75 R_{T}$ and longer. The above mentioned exception is for a $0.75 \mathrm{~h} / R_{T}$ with a $0.125 R_{T}$ inlet cone length. This case however provides significantly poorer performance than all other tests.

It should be noted that there does seem to be a natural transition from a very sharp hump shape to the curved parabolic shaped inlet cone. This is generally affected by inlet cone length. The hub-to-tip ratio seems to have little effect on the type of shape found except for the single exception at $0.75 \mathrm{~h} / R_{T}$. 
114 Computer Aided Optimum Design in Engineering XII
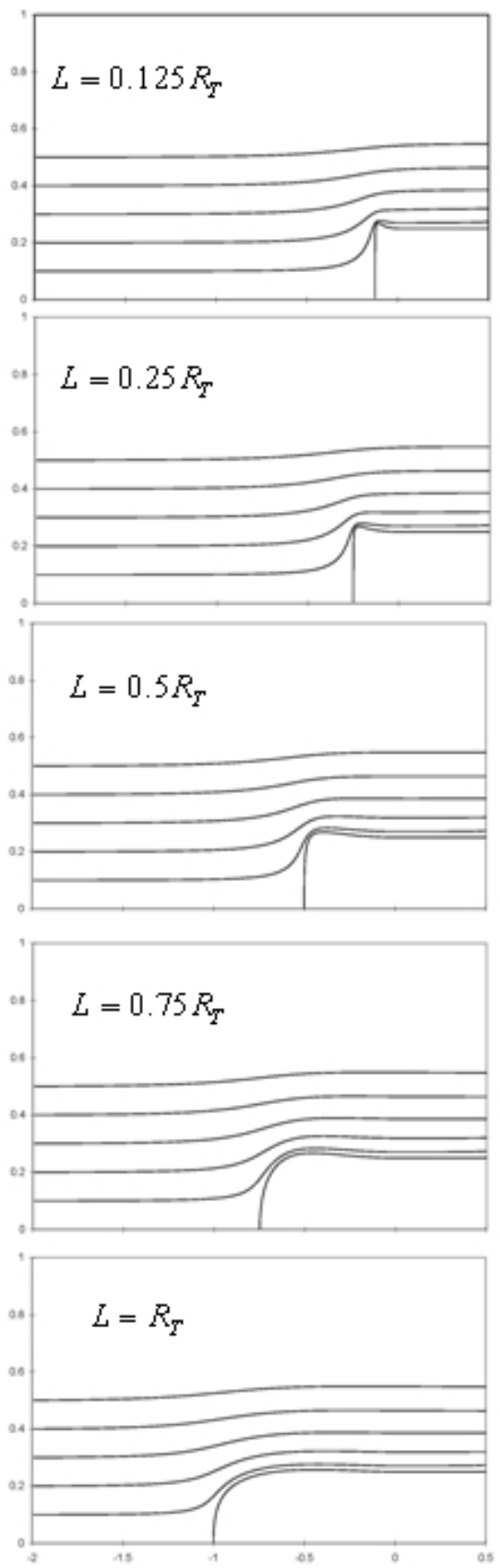

Figure 2: The effect of hub length for a $h / t=0.25$ on hub shape and streamlines. 

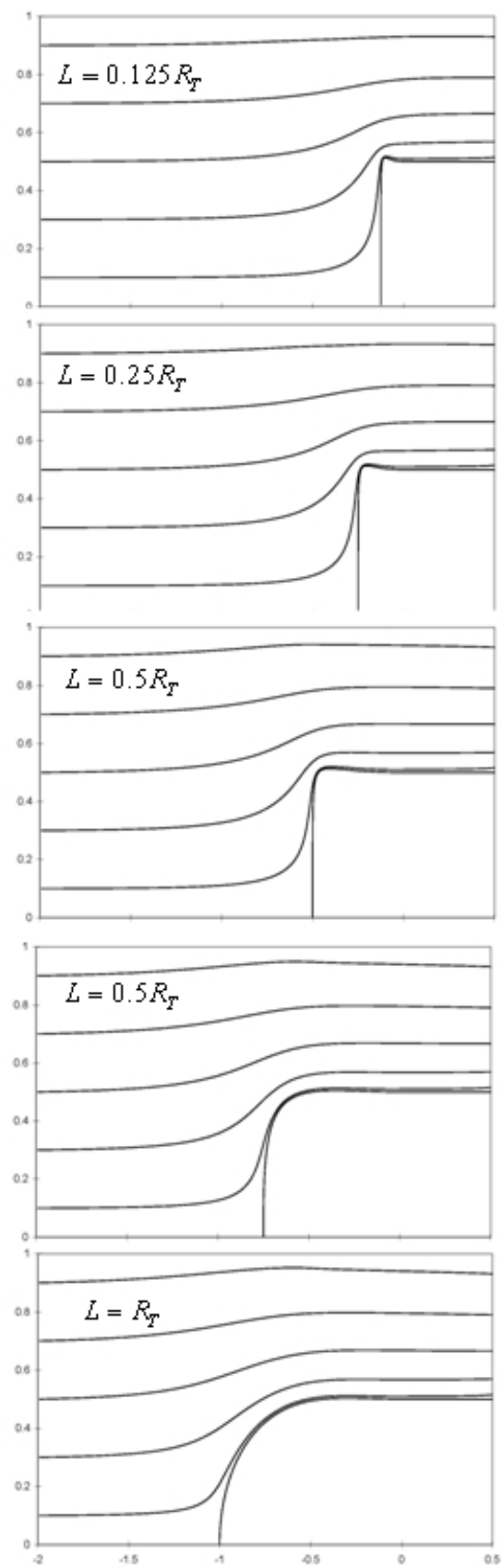

Figure 3: The effect of hub length for a $h / t=0.5$ on hub shape and streamlines. 
116 Computer Aided Optimum Design in Engineering XII
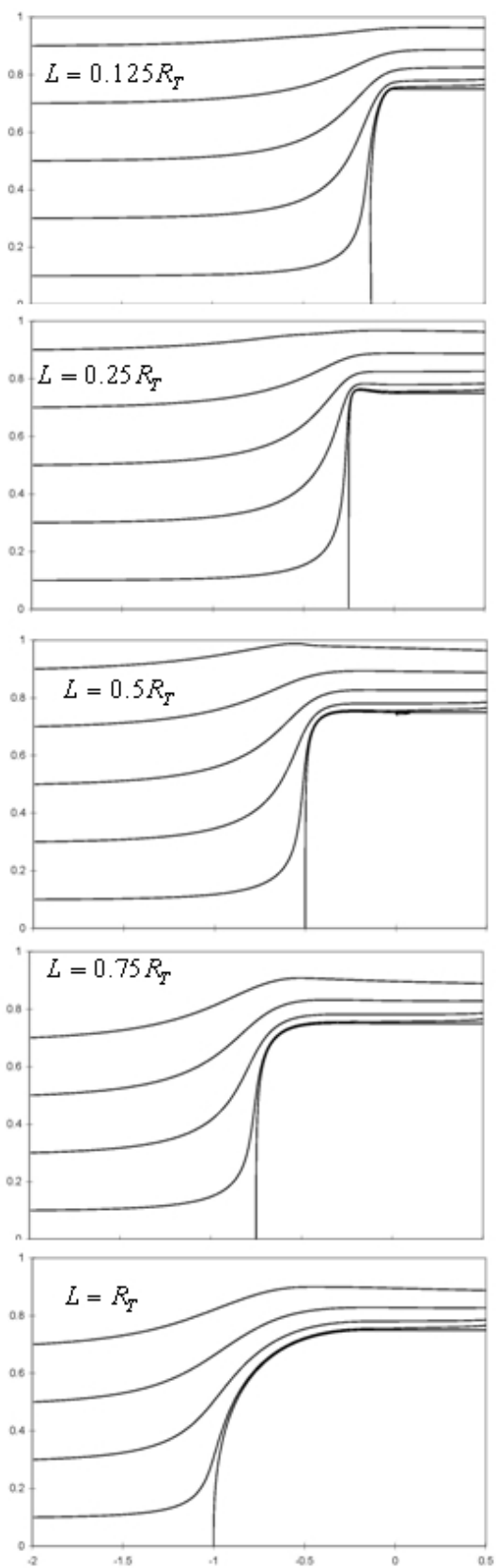

Figure 4: The effect of hub length for a $h / t=0.75$ on hub shape and streamlines. 


\section{Conclusions and recommendations}

The most obvious conclusion is that the use of Bezier curves to model the surface provides an exceptional design tool. Not only is the surface controlled by only a few control points that can be used as design variables but the generated surface is guaranteed to be smooth and not oscillate away from the defining points.

In comparison to the other minimization methods tested, the downhill simplex method in multidimensions is far superior in terms of efficiency. There is still a small concern regarding the minimization to a local minimum instead of a global minimum. However, due to the geometrical constraints placed on the design variables and the extensive testing with this problem it is reasonable to assume that the downhill simplex method is locating the global minimum. The very simple objective function used in this problem was developed to force the velocity distribution towards a uniform profile. Subsequent testing proved the objective function to be highly successful in this capacity.

The main question regarding the optimization program is whether or not the surface vorticity panel method is adequate or not. From the numerous tests done using this method the panel method is shown to produce reasonable qualitative results for problems that do not exhibit extreme geometries such as regions of obvious separation and discontinuous surfaces.

Two main types of inlet cones were found using the optimization code. Fans with short inlet cones, less than $0.75 h / R_{T}$, typically displayed the first type. Fans with longer inlet cones generally made use of the second type of inlet cone. The first type of inlet cone has a characteristic hump near the front of the inlet cone and a very blunt face. The second type of inlet cone is generally a parabolic shaped inlet cone. There does seem to be a natural transition between the two types as the inlet cone length is increased.

Two important conclusions can be made regarding the effects of cone length and hub-to-tip ratio on the velocity distribution. First, the length of the inlet cone has a significant effect on the velocity distribution for inlet cones shorter than $0.75 \mathrm{~h} / R_{T} \mathrm{~m}$ for the test case. For lengths greater than this the effect is limited and therefore there is no reason to attempt to improve the efficiency by drastically increasing the length of the inlet cone.

There are many possibilities for further work involving improvements to the program and further testing and verification of the program. The main areas that could possibly be improved upon are the flow solver and the minimization method.

One of the most important improvements that could be made to this program is the addition of a boundary layer solver. This would provide a more realistic approximation to the flow and the necessary corrections for the case of separated flow. While the inclusion of a boundary layer solver would increase the accuracy of the flow solver, and hence the final solution, it would significantly increase the complexity of the computer code and the computational effort required to produce a solution. 
One important step in the development of this program remains to be done, experimental verification. The results from this program should be tested experimentally before the program is used for practical applications in order to increase the confidence in the final solution.

One final improvement that may be of practical interest is the addition of an efficiency calculation before and after the optimization is performed. This would provide an estimate for the overall improvement in the total fan efficiency. This improvement, while being of practical interest would require substantial development work due to the need to model the three-dimensional effects of the fan blades.

\section{References}

[1] Bender, J.R., Aerodynamic Shape Optimization of Axial Flow Fans, M.Sc. Thesis, University of Manitoba, Winnipeg, Canada, 1997.

[2] Lewis, R.I., Vortex Element Methods For Fluid Dynamic Analysis of Engineering Systems, Cambridge University Press, Cambridge, pp.1-43, 146-176, 1991.

[3] Newman, W.M., and Sproull, R.F., Principles of Interactive Computer Graphics" McGraw-Hill, Tokyo, Japan, pp.315-318, 1981.

[4] Paulidis, T., Algorithms For Graphics and Image Processing, Computer Science Press, Rockville, MD., pp.221-230, 1982.

[5] Press, W.H., Vetterling, W.T., Teukolsky, S.A., and Flannery, B.P., Numerical Recipes in FORTRAN: the art of scientific computing, $2^{\text {nd }}$ ed., Cambridge University Press, Cambridge, pp.34-40, 51-63, 124-133, 206209, 254-261, 387-448, 1992.

[6] Nelder, J.A. and Mead, R., "A Simplex Method for Function Minimization," Computer Journal, Vol. 7, pp.308-313, 1965. 